\title{
The diagnostic role of endoscopic ultrasonography, magnetic resonance imaging and computed tomography in the assessment of pancreatic cystic lesions
}

Beyza Olcay Öztürk¹, Nergiz Ekmen², Mehmet Cindoruk², Güner Kılıç², Ali Karataş², Hadi Sasani ${ }^{3}$, Murat Uçar ${ }^{4}$

1Department of Internal Medicine, Ilgaz State Hospital, Çankırl, Turkey

2Department of Gastroenterology, Gazi University, Faculty of Medicine, Ankara, Turkey

3Department of Radiology, Tekirdăg Namık Kemal University, Faculty of Medicine, Tekirdag, Turkey

4Department of Radiology, Gazi University, Faculty of Medicine, Ankara, Turkey

Received: 2021-05-05.

Accepted: 2021-07-10

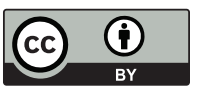

This work is licensed under a Creative Commons Attribution 4.0 International License

J Clin Med Kaz 2021; 18(4):69-74

Corresponding author:

Nergiz Ekmen.

E-mail: dr_nergisekmen@hotmail.com;

ORCID: 0000-0002-7921-3169

\section{Abstract}

Aim: To reveal the malignancy rates of pancreatic cystic lesions and success rates of Computed tomography (CT), magnetic resonance imaging (MRI) and endoscopic ultrasonography (EUS) in differentiating between malignant vs non-malignant cystic lesions of the pancreas.

Methods: In this study, 138 patients were included. CT, MRI and EUS and pathology reports of the related patients were scrutinized and descriptive statistical methods were used. Sensitivity and specificity values of CT, MRI and EUS were calculated by taking the postoperative histopathology results as the gold standard.

Results: In assessment the data of patients with neoplastic or nonneoplastic pancreatic cysts, the highest sensitivity, specificity and diagnostic accuracy in the malignant-benign distinction of the lesions was determined in CT. Although EUS had similar sensitivity and diagnostic accuracy rates as CT, its specificity was considered to be lower than CT. MRI had lower sensitivity, specificity and diagnostic accuracy rates compared to these modalities (EUS and CT). The sensitivity, specificity and diagnostic accuracy of EUS guided fine-needle aspiration biopsies were found to be quite low than other diagnostic modalities.

Conclusion: While CT and EUS showed high correlation with each other in terms of overall diagnostic compliance, there was a moderate correlation between MRI and EUS.

Key words: pancreas, cyst, neoplasm, ultrasonography, computed tomography, magnetic resonance imaging

\begin{abstract}
Introduction
Pancreatic cysts (PC) are detected incidentally. Their prevalence in asymptomatic patients is between $2.4 \%$ and $13.5 \%$, and its incidence increases with age [1]. The increase in the number of PC over the years is associated with an increase in the number of abdominal imaging. Concern about $\mathrm{PC}$ is due to the transformation of some cysts into ductal adenocarcinoma [2]. Considering all PCs, although they are thought to be at low-risk malignancy [3], the rate of malignancy may be up to $42 \%$ in some PCs [4]. Therefore, diagnosis and follow-up of $\mathrm{PC}$ is important.
\end{abstract}

Frequency of PC is $2.4-2.6 \%$ and increasing with age. It is known that the frequency of diagnosis of PCs is increasing due to the widespread use of cross-sectional imaging methods $[1,5]$. PCs can be classified mainly as inflammatory fluid deposits, neoplastic cysts, nonneoplastic cysts, mucinous or non-mucinous cysts. Cysts with potential for malignancy are intraductal papillary mucinous neoplasia (IPMN), mucinous cystic neoplasia (MCN), solid pseudopapillary tumor (SPPN) and neuroendocrine tumors (NET) of the pancreas.

Seventeen percent of PCs in asymptomatic patients were defined as in situ and ductal adenocarcinoma 
and $42 \%$ as premalignant lesions. Twenty-eight percent of asymptomatic cysts were evaluated as $\mathrm{MCN}$, followed by $27 \%$ IPMN, $17 \%$ serous cystadenoma (SCA), and $2.5 \%$ as ductal adenocarcinoma [6].

In this study, we aimed to reveal the malignancy rates of PC lesions and the success rates of imaging modalities such as endoscopic ultrasonography (EUS), computed tomography (CT), and magnetic resonance imaging (MRI) in differentiating between malignant and non-malignant cystic lesions of the pancreas.

\section{Materials and methods Patient selection}

The ethics commission of the institution approved the current study protocol (No: 91610558-604.01.02).

EUS data of 2500 patients over the age of 18 years between January 2015 and April 2019 were examined in the study. Out of 170 patients with pancreatic cystic lesions, 32 patients without CT or MRI were excluded from the study and 138 patients were included. The data of the patients were analyzed by reviewing fasting blood glucose, carcinoembryonic antigen (CEA) and amylase levels in cyst fluid, imaging (CT, MRI, EUS) findings retrospectively.

\section{CT protocol}

CT acquisitions were performed on a dual source $\mathrm{CT}$ device (SOMATOM Force, Siemens Healthineers, Munich, Germany). For abdominal CT examination, all patients were given a contrast agent (120 ml non-ionic contrast material; iohexol, Omnipaque; GE Healthcare, Cork, Ireland) orally and intraveneously. For IV injection of the contrast material a mechanical injector was used at a flow rate of 3-5 ml/sec. Abdomen and pancreas were evaluated in the early arterial portal and hepatic phases. CT scanning parameters were as following; 100-300 mAs, 100-120 $\mathrm{kV}$, 64x0.6 collimation, gantry rotation time: 0.5 second, pitch value: 0.8 , slice thickness: $3 \mathrm{~mm}$, reconstruction spacing $3 \mathrm{~mm}$, scan direction: craniocaudal.

\section{MRI protocol}

MRI acquisitions were performed on 3T MR scanner (Verio 3T MR scanner, Siemens Healthineers, Munich, Germany). Contrast agent was not used for abdominal MR examination. After 4 hours of fasting, imaging was performed using T2 sequence (axial plane, TR/TE: 2000/96 msec, Flip angle: 150, NEX:1, phase direction: anterior- posterior, echo tain length: 168, matrix: 320x224, field of view: $380 \mathrm{~mm}$, fat saturation: SPAIR, breath-hold respiration), and MRCP 3D sequence (coronal plane, TR/TE: 2400/719 msec, Flip angle: variable, NEX:2, phase direction: right- left, echo tain length: 101, matrix: 380x380, field of view: $300 \mathrm{~mm}$, fat saturation: SPAIR, navigator respiration).

\section{EUS protocol}

EUS evaluation was done with Olympus EVIS EXERA III CV-190 ecoendoscopic device by scanning from the duodenum up to the gastric corpus. The procedure was performed on an empty stomach with at least 8 hours fasting (starting from the midnight 00:00) before the procedure. Sedation (midazolam, propofol, fentanyl) and analgesia were given before EUS examination.

In EUS, the nature of the lesion (solid or cystic), number and size, localization, morphological features (septation, lobulation, wall thickness, presence of mural nodule, calcification, connection with pancreatic duct and channel width), presence of lymph node, invasion of vascular structures and potential peripheral organ invasion were evaluated.

\section{EUS-guided cystic lesion aspiration}

For aspiration process, 19-22 Gauge needle and a Linear EUS (Olympus EVIS EXERA III CV-190) device was used.

The needles were mostly inserted into the cyst only once and the aspiration process was continued until the cyst disappeared as much as possible. In the patients having cysts with mural nodules or increased wall thickness, the cysts were re-entered after aspiration of the cyst fluid and aspiration from the wall or wall nodule.

PC fluid was primarily evaluated for cytopathological examination. If the aspirated fluid was above one cc, tumor markers such as CEA and CA 19-9 and amylase were examined.

\section{Cytopathological evaluation}

The material obtained from the PC fluid was evaluated by expert cytologists. Staining with hemotoxylin-eosin, Papanicolaou (PAP) and diff quick.

\section{PCs evaluation}

The World Health Organisation (WHO) classification was used in assessment of PCs which is categorized in to three main groups including: benign, premalignant, and malignant [8]. The major histologic subtypes are serous cystic neoplasms (SCN), MCN, IPMN) and SPPN [7].

\section{Statistical analysis}

Statistical package program (SPSS version 22 for Windows; SPSS inc, Chicago) was used for statistical analysis. Descriptive statistical methods were used for all features of pancreatic cysts. Continuous data were summarized as mean \pm standard deviation and median (minimum-maximum), and categorical data were summarized with frequencies and percentages. For continuous variables without normal distribution, Mann-Whitney $U$ test was applied in independent groups and $\mathrm{p}<0.005$ value was considered as significant. Specificity, sensitivity, positive predictive value (PPV) and negative predictive value (NPV) of CT, MRI and EUS were calculated by taking the postoperative histopathological results as gold standard.

\section{Results \\ Demographic data}

A total of 138 patients (57 males, $41.3 \%$; 81 females, $58.7 \%$ ) with neoplastic or non-neoplastic PC aged between 19- 85 years (mean $59.30 \pm 13.06$ years) were included in the study. While $56.5 \%(n=78)$ of the patients had symptoms such as abdominal pain, jaundice, weight loss and vomiting, 43.5\% $(n=60)$ were followed asymptomatically. Sixty-four patients (46.4\%) had concomitant type 2 diabetes mellitus (DM).

\section{Radiological and gastroenterological imaging findings of patients}

Of the 138 patients who underwent EUS, $83.3 \%(n=115)$ had only MR results and 55.8\% (n:77) had CT results. While the most common detected lesion in MR was the side branch IPMN (38.2\%, n:=44), the most common lesions detected in CT were solid cystic degeneration $(28.5 \%, \mathrm{n}=22)$ and unspecified cystic lesion $(28.5 \%, \mathrm{n}=22)$; the most frequently detected lesion 
in EUS was solid tumor with cystic degeneration $(21.7 \%, \mathrm{n}=30)$.

PC localizations are shown in Figure 1. The most common localization was at the pancreatic head $(32.6 \%, n=45)$. The neoplasia rates determined by $\mathrm{CT}, \mathrm{MR}$ and EUS are shown in Figure 1. The relationship between the neoplastic and nonneoplastic lesions detected by fine-needle aspiration biopsy (FNAB) and surgical materials; and cyst sizes detected in CT, MR and EUS were examined. There was no statistically significant difference between cyst size and neoplasia detected by any imaging method ( $p>0.05$ ) (Table 1$)$.

Of 138 patients with EUS, peripancreatic lymphadenopathy in $13.8 \%(\mathrm{n}=19)$ and pancreatic canal dilatation in $70 \%(\mathrm{n}=98)$ was found. There was no significant relationship between the presence of peripancreatic lymphadenopathy and malignancy $(\mathrm{p}>0.005)$.
Figure 1 - Figure 1 shows the neoplasia rates determined by CT, MR and EUS.

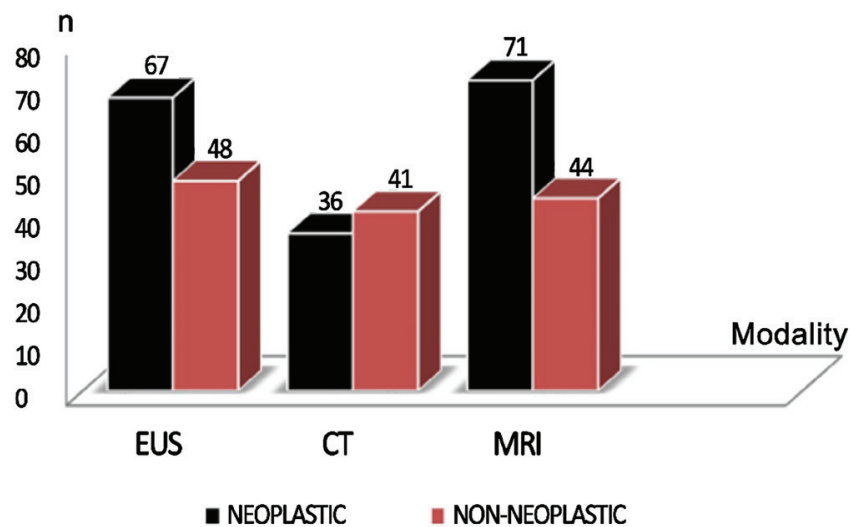

\begin{tabular}{|c|c|c|c|}
\hline Diagnosis & CT, n (\%) & MRI, n (\%) & EUS, n (\%) \\
\hline Simple cystic lesion & $0(0)$ & $16(13.9)$ & $6(4.3)$ \\
\hline Solid tumor with cystic degeneration & $22(28.5)$ & $16(13.9)$ & 30 (21.7) \\
\hline Lymphocele & $1(1.2)$ & $1(0.8)$ & $1(0.7)$ \\
\hline Unspecified cystic lesion & $22(28.5)$ & $5(4.3)$ & $26(18.8)$ \\
\hline Pseudocyst & $17(22)$ & $20(17.3)$ & $21(15.3)$ \\
\hline Walled off necrosis & $1(1.2)$ & $2(1.7)$ & $2(1.4)$ \\
\hline Serous cystadenoma & $2(2.5)$ & $5(4.3)$ & $5(3.6)$ \\
\hline Mucinosis cystadenoma & $1(1.2)$ & $3(2.6)$ & $4(2.9)$ \\
\hline Mucinous cystic adenocarcinoma & $1(1.2)$ & $2(1.7)$ & $4(2.9)$ \\
\hline Minor branch IPMN & $8(10.3)$ & $44(38.2)$ & $26(18.8)$ \\
\hline Main channel IPMN & $1(1.2)$ & $1(0.8)$ & $13(9.4)$ \\
\hline Pancreatic adenocarcinoma & $1(1.2)$ & $0(0)$ & $0(0)$ \\
\hline Variable & CT $(\mathrm{mm})$ & MRI (mm) & EUS (mm) \\
\hline Non-neoplastic, $\mathrm{n}$ & 24 & 27 & 33 \\
\hline Median (min-max) & $34.5(10-107)$ & $30(9-90)$ & $30(5-90)$ \\
\hline Neoplastic, $\mathrm{n}$ & 13 & 17 & 24 \\
\hline Median (min-max) & $30(21-70)$ & $35(6-80)$ & $36(4-70)$ \\
\hline $\mathrm{P}$ & 0.491 & 0.426 & 0.903 \\
\hline Variable & Amylase & CA $19-9$ & CEA \\
\hline Non-neoplastic, $\mathrm{n}$ & 16 & 6 & 17 \\
\hline Median (min-max) & $44.72(0.10-78830)$ & $1020.03(3.90-20460)$ & $650(5-112445)$ \\
\hline Neoplastic, $\mathrm{n}$ & 5 & 2 & 7 \\
\hline Median (min-max) & $747(45.98-44700)$ & $20330(20200-20460)$ & $2989(8.50-127375)$ \\
\hline $\mathrm{P}$ & 0.032 & 0.131 & 0.589 \\
\hline
\end{tabular}

\section{Comparison of cyst features detected by CT,} MRI and EUS with histopathology

The sensitivity and specificity values of patients considered as the gold standard of surgical pathology for the detection of neoplasia, are summarized in Table 2.

The sensitivity and specificity of the modalities in detecting neoplasia were $90.9 \%$ and $75 \%$ for CT, $71,4 \%$ and $66,7 \%$ for MRI and $89,5 \%$ and $50 \%$ for EUS, respectively.

In comparison of FNAB results $(\mathrm{n}=16)$ of the same patients with the surgical biopsy results, the FNAB sensitivity and the specificity were found to be $42.9 \%$ and $50 \%$, respectively.

Diagnostic accuracy rate NPV and PPV of the imaging methods are summarized in Table 2. The diagnostic accuracy rate for CT was $86.6 \%$ and NPV, PPV, sensitivity and specificity were higher in other examination methods.

The sensitivity and specificity, diagnostic accuracy rates, predictive values of the patients with regard to differentiating mucinous-non-mucinous lesions by considering the surgical pathology as the gold standard are summarized in Table 2. EUS had a high sensitivity $(50 \%)$ and diagnostic accuracy $(73.9 \%)$ in detecting mucinous tumors compared to CT and MRI. 


\begin{tabular}{|c|c|c|c|c|c|}
\hline \multicolumn{6}{|c|}{ Neoplastic Sensitivity and specificity Diagnostic accuracy and predictive values } \\
\hline Modality & $\begin{array}{l}\text { Diagnostic accuracy } \\
\text { rate } \%\end{array}$ & NPD \% & PPD \% & Sensitivity \% & Specificity $\%$ \\
\hline CT & 86.6 & 75 & 90.9 & 90.9 & 75 \\
\hline MRI & 70.5 & 33.3 & 90.9 & 71.4 & 66.7 \\
\hline EUS & 82.6 & 50 & 89.5 & 89.5 & 50 \\
\hline EUS-guided FNAB & 43.7 & 11.1 & 87.5 & 42.9 & 50 \\
\hline \multicolumn{6}{|c|}{ Sensitivity specificity, diagnostic accuracy, and predictive rates of modalities in detecting mucinous lesions } \\
\hline CT & 73.3 & 91.7 & 0 & 0 & 78.6 \\
\hline MRI & 70.5 & 78.5 & 33.3 & 25 & 84.6 \\
\hline EUS & 73.9 & 88.2 & 33.3 & 50 & 78.9 \\
\hline \multicolumn{6}{|c|}{ Overall compliance assessment of modalities } \\
\hline Modalities & kappa & $\mathrm{p}$ & & & \\
\hline EUS and CT & 0.613 & $0.0001^{*}$ & & & \\
\hline EUS and MRI & 0.638 & $0.0001^{*}$ & & & \\
\hline CT and MRI & 0.487 & $0.0001^{*}$ & & & \\
\hline
\end{tabular}

* Pearson chi square test was used.

- FNAB: Fine- needle aspiration biopsy

\section{Considering the modalities in terms of general diagnostic compliance}

MRI and EUS were moderately compatible (58.2\%, kappa $=0.406)$, CT and EUS were well compatible $(68.8 \%$, kappa $=0.629), \mathrm{CT}$ and MRI were found to be moderately compatible $(59 \%$, kappa $=0.517)$.

The overall compliance for MRI and EUS was found to be $82.6 \%$ in distinguishing neoplasia. Additionally, in evaluating the consistency between the two modalities (MRI and EUS) with the kappa test, a statistically significant and good agreement was observed (kappa 0.628 and $\mathrm{p}<0.001$ ).

The overall compliance for CT and EUS in distinguishing neoplasia was $80.5 \%$. In assessment the consistency between these modalities with the kappa test, a statistically significant and good agreement was observed (kappa 0.613 and $\mathrm{p}<0.001$ ).

The overall compliance for CT and MRI in distinguishing neoplasia was found to be $74.1 \%$. In evaluation the consistency between the two modalities with the kappa test, a statistically significant and good agreement was observed (kappa 0.487 and $\mathrm{p}<0.001)$ (Table 2).

\section{EUS guided FNAB pancreatic cyst fluid analysis}

In the examination of PC fluid with EUS-FNAB and the evaluation of CEA, CA19-9 and amylase levels; the median CEA level (2989 U/Ml) and CA19-9 (20330 U/Ml) in neoplastic lesions were measured higher than non-neoplastic lesions, however, the difference between them was not statistically significant $(\mathrm{p}>0.05)$ (Table 1).

A statistically significant difference was found between neoplastic and non-neoplastic lesions only with the level of cyst fluid amylase and non-neoplastic lesions had higher amylase levels $(p=0.032)$ (Table 1). CEA median values of cyst fluid in $\operatorname{IPMN}(\mathrm{n}=8)$ and $\mathrm{MCN}(\mathrm{n}=4)$ were determined as 146.23 (range: 8.5-603) and 772.5 (range: 15.7-78830), respectively and no significant difference was found $(\mathrm{p}=0.126)$.

\section{Discussion}

Pancreatic cysts (PCs) are diagnosed much more frequently nowadays with the increasing use of cross-sectional imaging methods in different diagnoses. Accurate diagnosis and typing of PCs is of great importance due to the risk of malignancy in some subtypes of PCs, the prevention of malignancy with treatment, or increasing the survival of patients by providing early diagnosis with follow-ups. The malignancy potential of PCs can be determined by typing with imaging methods, clinical, biochemical markers and cytological findings.

In the current study, $58.7 \%(\mathrm{n}=81)$ of PCs were found in females and the median age was 60 , the data which is compatible with the literature [8-10]. It was observed that $56.5 \%(n=78)$ of 138 PC patients were diagnosed symptomatically and remaining patients $(43.5 \%, \mathrm{n}=60)$ were completely asymptomatic and PC was detected incidentally. In the study by Fernandez et al., 134 (63\%) of 212 cases were found symptomatic [6]. In another study by Attasaranya et al, similar to the study of Fernandez et al, approximately $2 / 3$ of the patients were reported to be symptomatic [9]. In a study conducted by Mizuno et al, DM was found to be significantly higher in patients with PCs [11]. In our study, the rate of DM was $46 \%$ and it was more common than the normal population.

In examining the relation of neoplastic and non-neoplastic lesions detected by EUS-FNA with cyst sizes in CT, MR and EUS, no relation was found in any imaging method. According to the literature data, although the risk of malignancy is increased in cysts over $30 \mathrm{~mm}$ [12], in some publications, this relationship has not been shown. In the study conducted by Spinelli et al., no connection between the cyst diameter and the risk of malignancy was shown, and a cyst in a small size did not mean of not being malignant [13].

In the present study, the most common location of PCs was the pancreatic head $(32.6 \%)$. Similarly as in most studies, the most common localization was the pancreatic head which this rate reaches up to $63 \%[6,10,14]$.

Although IPMN was not defined in the literature before 1980, approximately $60 \%$ of incidentally detected PCs were 
mucin-secreting neoplasias (MCN and IPMN), 30\% benign non-neoplastic cysts, $5 \%$ SCN, $2 \%$ cystic NET and $3 \%$ have been shown to be pseudocysts [15]. In our study, PCs detected radiologically were found to be approximately $43 \%$ mucinous cystic lesions, about $40 \%$ non-neoplastic cysts, about $3 \% \mathrm{SCN}$, and about $14 \%$ pseudocyst. The difference between EUS-FNAB and postoperative pathology results was thought to be primarily due to lack of on-site biopsy.

In a study by Sperti et al., the sensitivity of $\mathrm{CT}$ in differentiating neoplastic and non-neoplastic lesions was $65 \%$, specificity $88 \%$, NPV $83 \%$ and PPV $73 \%$ and diagnostic accuracy rate was found to be $80 \%$ [16]. Although the diagnostic accuracy rates were similar in our study, the sensitivity and specificity rates may have resulted differently from our study due to the fewer patients included in the study by Sperti et al.

In the study by Lee et al, according to the radiologist report differentiating neoplasia and non-neoplastic lesion the sensitivity was ranging between $65.4-76.9 \%$, specificity was ranging between 58.3-88.9\%, NPV was ranging between 74.3$78 \%$, PPV was ranging between $57.1-81 \%$, and diagnostic accuracy was ranging between 55.6-76.2\%. Similar values were found in our study.

In the differentiation of EUS neoplastic and non-neoplastic lesions, Gerke et al. determined the sensitivity rate as $48-87 \%$, specificity as $49-80 \%$ and diagnostic accuracy rate as $65-67 \%$ [16]. On the other hand, in the study of Sedlack et al., the rates of differentiating benign lesions from malignant lesions in EUS was determined as followings: sensitivity $91 \%$, specificity $60 \%$ and diagnostic accuracy rate $82 \%$, respectively [17]. Since EUS is an operator-dependent procedure, there are large differences in the sensitivity and specificity of EUS. The values determined in our study were similar to those of Sedlack et al [15].

In a study of confirmed pathologically having PCs of 108 patients [18], EUS with or without FNA had a significantly increased accuracy for diagnosing PCs with highest sensitivity $(81.6 \%)$ and specificity $(86.1 \%)$ compared to CT and MRI. EUS was found superior to CT in the characterization of PCs in small $(<3 \mathrm{~cm})$ lesions but, it was superior to MRI in large $(>3 \mathrm{~cm})$ lesions. In our study CT was found to have the highest sensitivity and specificity compared to other modalities. There was no statistically significant difference between cyst size and neoplastic pathology detected by the modalities.

In a study similar to the results of our study conducted by Lee et al in 34 PCs patients who underwent three imaging modalities three months before surgery, they found that PCs size detected by EUS tended to be read smaller in tail portion, while it was not in CT and MRI. The size on EUS was estimated to be smaller than on pathology when it was more than $4 \mathrm{~cm}$ [19]. The measurement in PCs compared to the relevant pathological size, EUS had the lowest agreement, but CT had the highest agreement. Thus, especially PCs located in the tail should be interpreted carefully when it is evaluated by EUS.
In a recent study, the sensitivity of EUS guided FNAB in distinguishing mucinous and non-mucinous cysts was $42 \%$ and specificity was $99 \%$ [20]. In two meta-analyzes, the sensitivity and specificity of EUS-FNA-based cytologies for neoplasticnon-neoplastic pancreatic cysts were $51 \%$ and $94 \%$, respectively $[21,22]$. Sensitivity and specificity can be increased in patients who have undergone cyst fluid aspiration due to insufficient cyst content or reported to have no atypical cells. Therefore, the presence of an on-site cytopathologist is very important. In the EUS-FNAB examination accompany by an on-site cytopathologist, there was a $10-15 \%$ increase in the diagnostic accuracy rate in the literature [23-25].

In our study, amylase level was higher in non-neoplastic lesions in cyst fluid. Since the amylase level is particularly high in pseudocysts, this is consistent with clinical data. In the study by Soyer et al., amylase level was higher in benign lesions, while it was lower in malignant lesions [26]. CEA and CA 19-9 levels in cyst fluid were similar in neoplastic and non-neoplastic lesions. In a metaanalysis performed by Cao et al, they found that CA199 level in cyst fluid had a high specificity, but low sensitivity in distinguishing benign lesions from malignant lesions. They concluded that the low levels of CA19-9 alone cannot indicate a cyst to be benign [27].

There were some limitations in the current study as follows: 1) Being a retrospective study and low number of operated patiens having postoperative histopathology results, 2) Although EUS-guided FNAB on-site pathological examination was performed in most patients, this procedure was not performed in some patients. We think that the low FNAB sensitivity can be due to this.

In conclusion, due to the more frequent diagnosis of cystic lesions of the pancreas and the risk of malignancy in some types of cysts, appropriate modalities (EUS, CT, MRI) should be selected according to the patient. In our study, CT was found to have higher sensitivity, specificity and diagnostic accuracy rates in differentiation of benign and malignant cysts compared to other modalities. Therefore, $\mathrm{CT}$, due to it non-invasive nature and being a more accessible modality, can be preferred in these patients. While CT and EUS showed high correlation with each other in terms of overall diagnostic compliance, there was a moderate correlation between MRI and EUS

We think that performing EUS guided-FNAB in conjunction with on-site cytopathologist will reveal the true quality of this method. Appropriate imaging method should be considered during the diagnosis, follow-up and treatment of patients.

Disclosures: There is no conflict of interest for all authors.

\section{Acknowledgements: None.}

Funding: None.

\section{References}

1. de Jong K, Nio CY, Hermans JJ, Dijkgraaf MG, Gouma DJ, van Eijck CH, et al. High prevalence of pancreatic cysts detected by screening magnetic resonance imaging examinations. Clin Gastroenterol Hepatol. 2010;8(9):806-11. doi: 10.1016/j.cgh.2010.05.017.

2. Elta GH, Enestvedt BK, Sauer BG, Lennon AM. ACG Clinical Guideline: Diagnosis and Management of Pancreatic Cysts. Am J Gastroenterol. 2018;113(4):464-79. doi: 10.1038/ajg.2018.14.

3. Gardner TB, Glass LM, Smith KD, Ripple GH, Barth RJ, Klibansky DA, et al. Pancreatic cyst prevalence and the risk of mucinproducing adenocarcinoma in US adults. Am J Gastroenterol. 2013;108(10):1546-50. doi: 10.1038/ajg.2013.103. 
4. Scheiman JM, Hwang JH, Moayyedi P. American gastroenterological association technical review on the diagnosis and management of asymptomatic neoplastic pancreatic cysts. Gastroenterology. 2015;148(4):824-48.e22. doi: 10.1053/j.gastro.2015.01.014.

5. Laffan TA, Horton KM, Klein AP, Berlanstein B, Siegelman SS, Kawamoto S, et al. Prevalence of unsuspected pancreatic cysts on MDCT. AJR Am J Roentgenol. 2008;191(3):802-7. doi: 10.2214/AJR.07.3340.

6. Fernández-del Castillo C, Targarona J, Thayer SP, Rattner DW, Brugge WR, Warshaw AL. Incidental pancreatic cysts: clinicopathologic characteristics and comparison with symptomatic patients. Arch Surg. 2003;138(4):427-3; discussion 433-4. doi: 10.1001/ archsurg.138.4.427.

7. Bosman FT, Carneiro F, Hruban RH, Theise ND. WHO classification of tumours of the digestive system: World Health Organization;2010.

8. Brugge WR, Lewandrowski K, Lee-Lewandrowski E, Centeno BA, Szydlo T, Regan S, et al. Diagnosis of pancreatic cystic neoplasms: a report of the cooperative pancreatic cyst study. Gastroenterology. 2004;126(5):1330-6. doi: 10.1053/j.gastro.2004.02.013.

9. Attasaranya S, Pais S, LeBlanc J, McHenry L, Sherman S, DeWitt JM. Endoscopic ultrasound-guided fine needle aspiration and cyst fluid analysis for pancreatic cysts. Jop. 2007;8:553-63.

10. Cho CS, Russ AJ, Loeffler AG, Rettammel RJ, Oudheusden G, Winslow ER, Weber SM. Preoperative classification of pancreatic cystic neoplasms: the clinical significance of diagnostic inaccuracy. Ann Surg Oncol. 2013;20(9):3112-9. doi: 10.1245/s10434-013-2986-6.

11. Mizuno S, Isayama H, Nakai Y, Yoshikawa T, Ishigaki K, Matsubara S, et al. Prevalence of Pancreatic Cystic Lesions Is Associated With Diabetes Mellitus and Obesity: An Analysis of 5296 Individuals Who Underwent a Preventive Medical Examination. Pancreas. 2017;46(6):801-805. doi: 10.1097/MPA.0000000000000833.

12. Hoffman RL, Gates JL, Kochman ML, Ginsberg GG, Ahmad NA, Chandrasekhara V, et al. Analysis of cyst size and tumor markers in the management of pancreatic cysts: support for the original Sendai criteria. J Am Coll Surg. 2015;220(6):1087-95. doi: 10.1016/j. jamcollsurg.2015.02.013.

13. Spinelli KS, Fromwiller TE, Daniel RA, Kiely JM, Nakeeb A, Komorowski RA, et al. Cystic pancreatic neoplasms: observe or operate. Ann Surg. 2004;239(5):651-7; discussion 657-9. doi: 10.1097/01.sla.0000124299.57430.ce.

14. Haghighi M, Sethi A, Tavassoly I, Gonda TA, Poneros JM, McBride RB. Diagnosis of Pancreatic Cystic Lesions by Virtual Slicing: Comparison of Diagnostic Potential of Needle-Based Confocal Laser Endomicroscopy versus Endoscopic Ultrasound-Guided FineNeedle Aspiration. J Pathol Inform. 2019;10:34. doi: 10.4103/jpi.jpi_32_19.

15. Garcea G, Ong SL, Rajesh A, Neal CP, Pollard CA, Berry DP, et al. Cystic lesions of the pancreas. A diagnostic and management dilemma. Pancreatology. 2008;8(3):236-51. doi: 10.1159/000134279.

16. Gerke H, Jaffe TA, Mitchell RM, Byrne MF, Stiffler HL, Branch MS, et al. Endoscopic ultrasound and computer tomography are inaccurate methods of classifying cystic pancreatic lesions. Dig Liver Dis. 2006;38(1):39-44. doi: 10.1016/j.dld.2005.09.023.

17. Sedlack R, Affi A, Vazquez-Sequeiros E, Norton ID, Clain JE, Wiersema MJ. Utility of EUS in the evaluation of cystic pancreatic lesions. Gastrointest Endosc. 2002;56(4):543-7. doi: 10.1067/mge.2002.128106.

18. Lu X, Zhang S, Ma C, Peng C, Lv Y, Zou X. The diagnostic value of EUS in pancreatic cystic neoplasms compared with CT and MRI. Endosc Ultrasound. 2015;4(4):324-9. doi: 10.4103/2303-9027.170425.

19. LeeYS, Paik KH, Kim HW, Lee JC, Kim J, Hwang JH. Comparison of Endoscopic Ultrasonography, Computed Tomography, and Magnetic Resonance Imaging for Pancreas Cystic Lesions. Medicine (Baltimore). 2015;94(41):e1666. doi: 10.1097/MD.0000000000001666.

20. Gillis A, Cipollone I, Cousins G, Conlon K. Does EUS-FNA molecular analysis carry additional value when compared to cytology in the diagnosis of pancreatic cystic neoplasm? A systematic review. HPB (Oxford). 2015;17(5):377-86. doi: 10.1111/hpb.12364.

21. Wang QX, Xiao J, Orange M, Zhang H, Zhu YQ. EUS-Guided FNA for Diagnosis of Pancreatic Cystic Lesions: a Meta-Analysis. Cell Physiol Biochem. 2015;36(3):1197-209. doi: 10.1159/000430290.

22. Suzuki R, Thosani N, Annangi S, Guha S, Bhutani MS. Diagnostic yield of EUS-FNA-based cytology distinguishing malignant and benign IPMNs: a systematic review and meta-analysis. Pancreatology. 2014;14(5):380-4. doi: 10.1016/j.pan.2014.07.006.

23. 23. Polkowski M, Larghi A, Weynand B, Boustière C, Giovannini M, Pujol B, et al; European Society of Gastrointestinal Endoscopy (ESGE). Learning, techniques, and complications of endoscopic ultrasound (EUS)-guided sampling in gastroenterology: European Society of Gastrointestinal Endoscopy (ESGE) Technical Guideline. Endoscopy. 2012;44(2):190-206. doi: 10.1055/s-0031-1291543.

24. Storch I, Jorda M, Thurer R, Raez L, Rocha-Lima C, Vernon S, et al. Advantage of EUS Trucut biopsy combined with fine-needle aspiration without immediate on-site cytopathologic examination. Gastrointest Endosc. 2006;64(4):505-11. doi: 10.1016/j.gie.2006.02.056.

25. T Turhan N, Aydog G, Ozin Y, Cicek B, Kurt M, Oguz D. Endoscopic ultrasonography-guided fine-needle aspiration for diagnosing upper gastrointestinal submucosal lesions: a prospective study of 50 cases. Diagn Cytopathol. 2011;39(11):808-17. doi: 10.1002/ dc. 21464.

26. S Soyer OM, Baran B, Ormeci AC, Sahin D, Gokturk S, Evirgen S, et al. Role of biochemistry and cytological analysis of cyst fluid for the differential diagnosis of pancreatic cysts: A retrospective cohort study. Medicine (Baltimore). 2017;96(1):e5513. doi: 10.1097/ MD.0000000000005513.

27. Cao S, Hu Y, Gao X, Liao Q, Zhao Y. Serum Carbohydrate Antigen 19-9 in Differential Diagnosis of Benign and Malignant Pancreatic Cystic Neoplasms: A Meta-Analysis. PLoS One. 2016;11(11):e0166406. doi: 10.1371/journal.pone.0166406. 